\title{
Treatment Decisions of World Health Organization Grade II and III Ependymomas in Molecular Era
}

\author{
Tae-Young Jung, M.D., Ph.D., ${ }^{1}$ Shin Jung, M.D., Ph.D., ${ }^{1}$ Hoon Kook, M.D., Ph.D., ${ }^{2}$ Hee-Jo Baek, M.D., Ph.D. ${ }^{2}$ \\ Departments of Neurosurgery, Pediatrics, ${ }^{2}$ Chonnam National University Hwasun Hospital, Chonnam National University Medical School, \\ Hwasun, Korea
}

Surgery and radiotherapy are mainstays of treatment for ependymomas (EPNs). Recent molecular subgrouping could be superior to histopathological grading for predicting the prognosis of patients with EPNs. Gross total resection is an effective treatment approach regardless of its locations or pathologic grades. Adjuvant therapeutic strategies could be decided based on molecular subgrouping with risk-stratification. Information of histologic-molecular biology is now providing clues to therapeutic insights.

Key Words : Ependymoma $\cdot$ Biology $\cdot$ Molecular medicine $\cdot$ Prognosis $\cdot$ Therapeutics.

\section{INTRODUCTION}

Ependymomas (EPNs) are neuroepithelial malignancies of the central nervous system (CNS) in children and adults, affecting children more frequently. They account for $1.8 \%$ of all primary CNS tumors and up to $10 \%$ of malignant brain tumors in childhood ${ }^{12}$. In children, $90 \%$ of EPNs occur intracranially, with two-thirds in the posterior fossa $(\mathrm{PF})$ and onethird in supratentorial location (ST). Ten percent of EPNs are located in spinal cord. For adult EPNs, the spinal cord is mainly affected. All EPNs are histologically similar but clinically and genetically different.

Major prognostic factors of EPNs are the extent of resection, age, the location of tumor, pathologic grade, and so on ${ }^{20,21)}$, although such factors remain controversial. Predicting tumor behavior and defining disease outcome based on histopathol- ogy and clinical characteristics remains suboptimal. As treatment, maximal safe resection followed by radiation therapy are the current standard treatment for pediatric $\mathrm{EPNs}^{19}$. In pediatric supratentorial EPNs, it could be acceptable to withhold radiotherapy if gross total resection with adequate margins has been achieved and the pathology is not anaplastic ${ }^{32)}$. In adult patients, observation after gross total resection of World Health Organization (WHO) II EPN and adjuvant radiation after subtotal resection are the standard practice ${ }^{1,5,17)}$. Radiation is recommended especially for cases of extensive residual disease and anaplastic EPN. The management of pediatric and adult patients with different grades of EPNs has not been standardized.

According to the WHO classification of CNS tumors, ependymal tumors are divided into subependymoma (SE; WHO grade I), myxopapillary EPN (MPE; WHO grade I), EPN

- Received : December 29, 2017 •Revised : February 4, 2018 •Accepted : February 14, 2018

- Address for reprints : Shin Jung, M.D., Ph.D.

Department of Neurosurgery, Chonnam National University Hwasun Hospital, Chonnam National University Medical School, 322 Seoyangro, Hwasun-eup, Hwasun 58128 , Korea

Tel : +82-61-379-7666, Fax : 82-61-379-7673, E-mail : sjung@chonnam.ac.kr

This is an Open Access article distributed under the terms of the Creative Commons Attribution Non-Commercial License (http://creativecommons.org/licenses/by-nc/4.0) which permits unrestricted non-commercial use, distribution, and reproduction in any medium, provided the original work is properly cited. 
(WHO grade II), EPN, v-rel avian reticuloendotheliosis viral oncogene homolog A (RELA) fusion-positive (WHO grade II or III), and anaplastic EPN (WHO grade III) ${ }^{14)}$. Grade I EPNs (SE and MPE) are relatively easier to distinguish. They occur mainly in adults. They are associated with favorable clinical outcome. Therefore, we focused on WHO grade II and III EPNs in this article. Classic EPN and anaplastic EPN are considered to correspond histologically to WHO grade II and III, respectively. A diagnosis of anaplastic EPN can be made when an ependymal tumor shows a high cell density and elevated mitotic count alongside widespread microvascular proliferation and necrosis. The goal of histological grading is to predict clinical prognosis and perform risk-stratification. However, the WHO criteria for EPN grading are not well defined yet ${ }^{14}$. The differentiation between II and III grades is often difficult with interobserver discordance ${ }^{8)}$. Furthermore, the relationship between grades and clinical survival has been controversial ${ }^{8,21}$. There have been genetic and clinical heterogeneities despite histological similarity. Recently, advanced genomic studies have been performed to decode EPN biology ${ }^{25}$. Molecular subgrouping along with the level of resection has been used as independent prognostic parameter for ependymal tumors ${ }^{25)}$. EPN grading may soon become a thing of the past. Treatment decisions for EPN should not be based on histologic grading only without molecular information ${ }^{23)}$.

Previous studies have demonstrated that there are location- specific molecular profiles and high degree of heterogeneity within locations ${ }^{2,24,25,27)}$. They are subsequently recognized to be biologically distinct in the supratentorial, posterior fossa, and spinal compartment of the CNS. Distinct entities of EPN have been defined by molecular profiling. The anatomical location of molecular subgroups is divided into three major anatomical compartments of the CNS : spine (SP), PF, and ST (Table 1). Based on associations with anatomical location, histology, and genetic alterations, nine distinct subgroups of ependymal tumors have been annotated : SP-SE, SP-MPE, SPEPN, PF-SE, PF-EPN-A, PF-EPN-B, ST-SE, ST-EPN-yesassociated protein 1 (YAP1), and ST-EPN-RELA ${ }^{25}$. With histopathologic grades, SP-MPE and SP-EPN have shown relatively good concordance ${ }^{25}$. However, there was no concordance between histopathological grade and PF or ST subgroup. Within these nine subgroups, there was a strong association of poor clinical behavior in ST-EPN-RELA and PF-EPN-A subgroups. Recent molecular classification of ependymal tumors has outperformed histopathological grade regarding clinical associations. Therefore, effective treatment decisions could be made based on histologic-molecular review with the risk-stratification. Information of histologic-molecular biology is now providing clues to therapeutic insights.

Table 1. Treatment decisions of WHO grade II and III ependymomas in molecular era

\begin{tabular}{|c|c|c|c|c|c|}
\hline Location & $\begin{array}{l}\text { Molecular } \\
\text { subgroups }\end{array}$ & Age & Genetics & Prognosis & Treatment \\
\hline \multirow[t]{2}{*}{ Supratentorial } & ST-EPN-RELA & Children, adults & $\begin{array}{l}\text { Aberrations Chr.11; } \\
\text { chromothripsis; } \\
\text { C11orf95-RELA fusion }\end{array}$ & Poor & $\begin{array}{l}\text { No enough evidence to recommend distinct } \\
\text { treatment approaches } \\
\text { Potential target : NF-kB inhibitor }\end{array}$ \\
\hline & ST-EPN-YAP1 & Children & $\begin{array}{l}\text { Focal aberrations Chr. 11; } \\
\text { YAP1 fusion }\end{array}$ & Good & $\begin{array}{l}\text { Recommend de-escalating therapy } \\
\text { Potential target: YAP1 inhibitor }\end{array}$ \\
\hline \multirow[t]{2}{*}{ Posterior fossa } & PF-EPN-A & Children ( $<5$ years) & $\begin{array}{l}\text { Balanced } \\
\text { CIMP-positive }\end{array}$ & Poor & $\begin{array}{l}\text { Surgery with adjuvant radiotherapy regardless of } \\
\text { the extent of resection in patients }>12 \text { months } \\
\text { of age } \\
\text { Potential target: DNA CpG methylation inhibitor }\end{array}$ \\
\hline & PF-EPN-B & $\begin{array}{l}\text { Children (5-16 years), } \\
\text { adults }\end{array}$ & $\begin{array}{l}\text { CIN } \\
\text { CIMP-negative }\end{array}$ & Good & $\begin{array}{l}\text { Surgery alone for gross total resection } \\
\text { Surgery with adjuvant radiotherapy for subtotal } \\
\text { resection }\end{array}$ \\
\hline Spinal cord & SP-EPN & Adults & CIN; NF2 mutation & Good & Recommend de-escalating therapy \\
\hline
\end{tabular}

WHO : World Health Organization, ST : supratentorial, EPN : ependymoma, RELA : v-rel avian reticuloendotheliosis viral oncogene homolog A, YAP1 : yes-associated protein 1, Chr. : chromosome, NF-KB : nuclear factor kappa-light-chain-enhancer of activated B cells, PF : posterior fossa, SP : spinal cord, CIMP : CpG island methylator phenotype, CIN : chromosomal instability 


\section{MOLECULAR SUBGROUPS OF WHO GRADE ॥ AND III EPENDYMOMAS}

\section{Supratentorial ependymomas (ST-EPN-RELA and ST-EPN-YAP1 subgroups)}

Supratentorial EPNs are divided into two distinct molecular subgroups: oncogenic mutually exclusive gene fusions involving either RELA or YAP1 ${ }^{25)}$. Although Illumina DNA methylation provides an indirect measure of molecular subgroup associated with a particular fusion, fluorescence in situ hybridization (FISH) can be used to identify and verify samples harboring C11orf95-RELA fusion ${ }^{15)}$. RNA-seq also provides an approach to identify C11orf95-RELA fusion as a discovery method to identify novel or less obvious fusion event. The immunohistochemistry can be performed for L1 cell adhesion molecule (L1CAM) protein, which is strongly associated with the C11orf95-RELA translocation ${ }^{27)}$. RELA fusion-positive EPNs, ST-EPN-RELA subgroup, has been recently added to the 2016 revision of the WHO classification of CNS tumors. It has dismal prognosis ${ }^{14)}$. All tumors with C11orf95-RELA fusion, mostly types 1 and 2, show telomerase activation. Activation of telomerase is a characteristic feature of ST-EPN-RELA. C11orf95RELA fusion is resulted from chromothripsis of chromosome 11q. No chromothripsis has been detected in tumor classified as ST-EPN-YAP1, even though YAP1 is also located on chromosome 11. Homozygous deletion of the CDKN2A/B locus has been frequently detected in ST-EPN-RELA tumors. It is associated with inferior prognosis ${ }^{12}$. C11orf95-RELA fusion proteins are translocated spontaneously to the nucleus to activate NF- $\kappa B$ signaling pathway ${ }^{25,27)}$. Cell cycle genes, cell migration genes, or genes involved in MAPK signaling are most active in ST-EPNRELA tumors. They are also active in PF-EPN-A tumors ${ }^{25)}$. The C11orf95-RELA fusion protein may be a potential therapeutic target in ST-EPN-RELA. YAP1 fusion-positive EPNs are defined as a distinct subgroup, ST-EPN-YAP1 $1^{25)}$. The most common fusion was YAP1-MAMLD1. Other fusion included YAP1FAM118B. Cyclic adenosine monophosphate/carbohydrate metabolism or dopamine signaling genes are active in ST-EPNYAP1 tumors ${ }^{25)}$.

ST-EPN-RELA occurs in 70\% of supratentorial tumors with median age of 8 years (range, $0-69$ years) ${ }^{25)}$. ST-EPN-YAP1 and ST-EPN-RELA are much more common in children. A significant portion of ST-EPN-RELA tumors are also found in adults. While patients in the ST-EPN-RELA subgroup comprised high- risk patients with 10-year overall survival (OS) of around 50\%, patients in the ST-EPN-YAP1 subgroup showed 100\% 10-year OS with 10-year progression-free survival (PFS) of around $20 \%{ }^{25}$.

\section{Posterior fossa ependymomas (PF-EPN-A and PF- EPN-B subgroups)}

PF-EPN-A and PF-EPN-B. They are classified based on gene expression and DNA cytosine-phosphate-guanine $(\mathrm{CpG})$ island methylation patterns using genome-wide methylation arrays $^{25)}$. Despite lacking a clear genetic driver mutation, PFEPN-A tumors have demonstrated widespread epigenomic alterations in DNA cytosine guanine dinucleotide island hypermethylation and global DNA hypomethylation. A higher extent of CpG island methylation was found in PF-EPN-A compared to that in PF-EPN-B ${ }^{16)}$. Therefore, PF-EPN-A referred to $\mathrm{CpG}$ island methylator phenotype (CIMP)-positive EPNs and PFEPN-B referred to CIMP-negative EPNs. For defining highrisk PF-EPN-A from low-risk PF-EPN-B, immunohistochemical staining of histone $\mathrm{H} 3$ lysine 27 trimethylation (H3K27me3) global reduction has been introduced as an economic, easily available, and adaptable method ${ }^{26)}$. Global reduction in the repressive mark $\mathrm{H} 3 \mathrm{~K} 27 \mathrm{me} 3$ is driven by aberrant DNA methylation. Laminin alpha 2 and neural epidermal growth factor like-2 stainings can be histological markers for PF-EPN-A and PF-EPN-B, respectively ${ }^{35)}$. It is also important to note that chromosome 1q gain has been shown to be an independent prognostic factor that occurs in a subset of PF-EPN-A, PFEPN-B, and ST-EPN-RELA tumors ${ }^{23)}$. Chromosome lq gain is strongly associated with telomerase reactivation in PF-EPN-A ${ }^{10)}$.

These subgroups are associated with distinct transcriptomic, genetic, epigenetic, and clinical features ${ }^{2}$. PF-EPN-A tumors predominantly occur in infants and young children younger than 5 years. They are located laterally in the posterior fossa with a relatively balanced genome, showing clinically aggressive behavior. PF-EPN-B tumors occur in adults and older children, showing better prognosis than PF-EPN-A tumors. PF-EPN-B tumors demonstrate wide-spread chromosomal aneuploidy ${ }^{35)}$. Although PF-EPN-A occurs primarily in infants while PF-EPN$\mathrm{B}$ is diagnosed primarily in adults, both PF-EPN-A and PFEPN-B occur at comparable frequencies in children between 5 and 16 years of age ${ }^{30)}$. Approximately $11 \%$ of adult patients have PF-EPN-A tumors. Routine subgrouping is necessary for these patients. In the pre-molecular era, age was an important prognostic factor for patients with posterior fossa EPN. However, 
poor prognosis of PF-EPN-A and excellent prognosis of PF-EPN$\mathrm{B}$ are independent of age at diagnosis ${ }^{30)}$.

\section{Spinal cord ependymomas (SP-EPN subgroup)}

SP-EPN subgroup occurs in spinal column, predominantly affecting adolescents and young adults. Neurofibromatosis type 2 (NF2) is frequently mutated. Such tumors show a high frequency of $22 \mathrm{q}$ homozygous loss ${ }^{25)}$. SP-EPNs with NF2 show more indolent clinical courses. Gross total resection has also shown benefit in SP-EPNs, with a progression free survival of $87 \%$ versus $57 \%$ in tumors that undergo subtotal resection ${ }^{3)}$. With a favorable prognosis, radiotherapy may not be necessary in patients after gross total resection of SP-EPNs.

\section{TREATMENT DECISION IN MOLECULAR ERA}

Even though the management of patients with EPNs lacks standardization, local control is very important to prevent recurrence as these tumors are locally invasive with low metastatic potential and leptomeningeal dissemination seen in a small minority of cases at diagnosis. Surgical resection and involvedfield radiation remain the mainstays of treatment for all EPNs.

Maximal safe resection, ideally gross total resection, is the mainstay of any treatment approach regardless of its locations or pathologic grades. The extent of surgical resection has been the most powerful prognostic marker in EPN known to be associated with PFS and $\mathrm{OS}^{12,21,34)}$. Even when treated with additional radiotherapy, patients with subtotal resection may remain at high risk for disease progression ${ }^{34)}$. An immediate postoperative brain magnetic resonance imaging, ideally within 24-48 hours after surgery, is recommended to determine the extent of removal. A second-look surgery has been proposed to maximize the extent of removal ${ }^{18)}$.

Postoperative radiation has been established as an effective modality for high-grade EPNs and for patients who are unable to undergo gross total resection. Even though the differentiation between EPN grade II and III is often difficult, there is a consensus that tumor resection should be followed by radiotherapy $^{8)}$. However, there is less clarity of postoperative radiotherapy for grade II EPN with gross total resection. Staging with imaging of the entire neuraxis and cerebrospinal fluid analysis is important in treatment planning. Cerebrospinal fluid (CSF) analysis should be delayed for a minimum of two weeks after the surgery to avoid confusing findings in the CSF. An involved-field radiotherapy is recommended unless there is a sign of tumor dissemination. The standard radiation dose to treat local EPN ranges from 54 to $59.4 \mathrm{~Gy}^{28,29)}$. Radiation margins around the tumor volume have also decreased from $2.0 \mathrm{~cm}$ to $1.0 \mathrm{~cm}$ without evidence of increased frequency of tumor relapse. The danger of radiotherapy has been focused in EPN patients younger than 3 years. However, young children with completely resected EPN in whom radiotherapy was delayed for two years had worse outcome, with 5-year survival of 38\%, lower than those in whom radiotherapy was delayed for one year, with 4 -year survival of $88 \%{ }^{30)}$. Therefore, delaying radiotherapy is not advisable for young children. Maximal safe resection and conformal radiotherapy could be defined as the standard care for patients older than 12 months of age ${ }^{23)}$. The role of craniospinal irradiation is reserved for patients with neuraxis dissemination.

The role of chemotherapy is less well established. Its effectiveness continues to be evaluated in numerous ongoing clinical trials. Chemotherapy has been used in infant and young children to spare normal developing nervous system from radiation strategies. Previous studies of chemotherapy have shown only limited activity against posterior fossa EPN. High-dose chemotherapy with autologous stem-cell support has resulted in 3-year event-free survival of less than 30\%, consistent with the survival of previous studies ${ }^{33)}$. In patients with recurrent disease, platinum-based chemotherapy might result in increased disease stability compared to treatment with nitrosourea-based regimens ${ }^{11}$. In adult patients with recurrent intracranial EPNs, cisplatinbased chemotherapy failed to prolong PFS or OS despite a higher objective response rate ${ }^{4}$. Temozolomide showed little efficacy for a small number of cases of adult recurrent intracranial $\mathrm{EPNs}^{6,31)}$. Collaborative EPN research network investigators have developed a novel clinical trial for adults with recurrent EPN, testing a combination treatment of lapatinib, a dual tyrosine kinase receptor inhibitor of epidermal growth factor receptor (EGFR/ERBB1) and ERBB2, and dose-dense temozolomide with the intention of depleting $\mathrm{O}^{6}$-methylguanine-DNA methyl-transferase. There was a statistical significant correlation between a better treatment response and a higher ERBB2 expression $^{9)}$. Elevated vascular endothelial growth factor expression has been observed in most cases of EPNs. It is associated with short survival and poor prognosis. Lapatinib combined with bevacizumab has been tested on children in a 
clinical trial. It showed a low response rate ${ }^{7}$. Clinical trials of chemotherapy area are still recruiting through cooperative groups. Up to date, there are no chemotherapeutic agents that can routinely be recommended.

In the molecular era, genetic characterization could be considered when formulating treatment plan with clinical prognostic factors. The 2017 consensus for the clinical management of EPNs suggests maximal resection and radiation for PF-EPN$\mathrm{A}$ in contrast to the potential for de-escalation of therapy for PF$\mathrm{EPN}^{2{ }^{23}}{ }^{2}$. A recent study has demonstrated that children with PF-EPN-A have the best outcomes when maximal safe surgical resection is combined with the first-line adjuvant radiotherapy. However, patients with PF-EPN-B showed good prognosis with surgical resection ${ }^{30}$. Both PF-EPN-A and PF-EPN-B could still benefit from increased extent of resection, with the survival rates being particularly poor for subtotal resection in both subgroups. In subgroup of PF-EPN-A, subtotal resection was highly predictive of a dismal PFS and OS compared to gross total resection ${ }^{30)}$. The benefit of first-line adjuvant radiation for patients with PF-EPN-A was limited for subtotal resection. Gross total resection should be attempted even in the molecular era ${ }^{30}$. A subset of patients with PF-EPN-B can be cured with surgery alone. Most PF-EPN-B patients have lower risk for relapse after gross total resection. Therefore, avoiding harmful radiotherapy could significantly improve long-term quality of life. In this subgroup, recurred cases may be treated successfully with repeat surgery and delayed delivery of radiation. These raise a possibility of future clinical trials to evaluate de-escalation therapy for PF-EPN-B patients. In supratentorial EPNs, there has been no enough evidence yet to recommend distinct treatment approaches for ST-EPN-RELA which has shown poor progno$\mathrm{sis}^{22,27)}$. Interestingly, it has been reported that the level of resection does not significantly affect the outcome within the STEPN-RELA-positive subgroup in a retrospective analysis for patient samples collected over a long period of time over 20 years ${ }^{25}$. ST-EPN-YAP1 subgroup is associated with favorable clinical outcome ${ }^{13,15}$. Clinical trials with de-escalating therapy could be recommended for this subgroup.

Molecular subtyping described above provides insights into tumor biology for potential therapeutic targets. PF-EPN-A is characterized by relatively increased DNA methylation compared to PF-EPN-B. Epigenetic modulating agents might be beneficial for patients with PF-EPN-A. Drugs that target DNA CpG methylation such as enhancer of zeste homolog 2 inhibi- tors or histone demethylase inhibitors mght be considered as potential therapeutic agents for treating PF-EPN-A and CIMPpositive $\mathrm{EPNs}^{16,30)}$. The complete lack of recurrent somatic single nucleotide variants implies that targeted therapy using small molecules directed against recurrent mutations is unlikely to be a successful strategy for patients with PF-EPN-A or PF-EPN-B. RELA fusion found in most supratentorial EPNs results in constitutive activation of NF- $\mathrm{KB}$, a potential therapeutic target ${ }^{27}$. NF- $\mathrm{\kappa B}$ or YAP1 inhibitors might have potential for supratentorial $\mathrm{EPNs}^{15)}$.

\section{CONCLUSION}

The management of EPN patients is still challenging. However, better understanding of its biology and molecular classification could not only improve current diagnostic accuracy and prognostication, but also provide future clinical trials. Recent molecular subgrouping of EPNs will help us make treatment decisions with the ultimate goal to improve the morbidity and mortality of children and adults with EPN.

\section{CONFLICTS OF INTEREST}

No potential conflict of interest relevant to this article was reported.

\section{INFORMED CONSENT}

This type of study does not require informed consent.

\section{- Acknowledgements}

This study was supported by a grant (2017R1A1A1A05001020) of Basic Science Research Program through the National Research Foundation of Korea (NRF) funded by the Ministry of Science, ICT, \& Future Planning, Republic of Korea.

\section{References}

1. Aizer AA, Ancukiewicz M, Nguyen PL, Macdonald SM, Yock TI, Tarbell $\mathrm{NJ}$, et al. : Natural history and role of radiation in patients with supratentorial and infratentorial WHO grade II ependymomas: results from a 
population-based study. J Neurooncol 115 : 411-419, 2013

2. Archer TC, Pomeroy SL : Posterior fossa ependymomas: a tale of two subtypes. Cancer Cell 20 : 133-134, 2011

3. Benesch M, Weber-Mzell D, Gerber NU, von Hoff K, Deinlein F, Krauss $J$, et al. : Ependymoma of the spinal cord in children and adolescents: a retrospective series from the HIT database. J Neurosurg Pediatr 6 : 137144, 2010

4. Brandes AA, Cavallo G, Reni M, Tosoni A, Nicolardi L, Scopece L, et al. : A multicenter retrospective study of chemotherapy for recurrent intracranial ependymal tumors in adults by the Gruppo Italiano Cooperativo di Neuro-Oncologia. Cancer 104 : 143-148, 2005

5. Chai YH, Jung S, Lee JK, Kim IY, Jang WY, Moon KS, et al. : Ependymomas: prognostic factors and outcome analysis in a retrospective series of 33 patients. Brain Tumor Res Treat 5 : 70-76, 2017

6. Chamberlain MC, Johnston SK : Temozolomide for recurrent intracranial supratentorial platinum-refractory ependymoma. Cancer 115 : 47754782, 2009

7. DeWire M, Fouladi M, Turner DC, Wetmore C, Hawkins C, Jacobs C, et al. : An open-label, two-stage, phase II study of bevacizumab and lapatinib in children with recurrent or refractory ependymoma: a collaborative ependymoma research network study (CERN). J Neurooncol 123 : 8591, 2015

8. Ellison DW, Kocak M, Figarella-Branger D, Felice G, Catherine $G$, Pietsch T, et al. : Histopathological grading of pediatric ependymoma: reproducibility and clinical relevance in European trial cohorts. J Negat Results Biomed $10:$ 7, 2011

9. Gilbert M, Yuan Y, Wani K, Wu J, Omuro A, Lieberman F, et al. : A phase Il study of lapatinib and dose dense temozolomide (TMZ) for adults with recurrent ependymoma: a CERN clinical trial. Neuro Oncol 16(suppl_5) : v13, 2014

10. Gojo J, Lötsch D, Spiegl-Kreinecker S, Pajtler KW, Neumayer K, Korbel P, et al. : Telomerase activation in posterior fossa group $A$ ependymomas is associated with dismal prognosis and chromosome 1q gain. Neuro Oncol 19 : 1183-1194, 2017

11. Gornet MK, Buckner JC, Marks RS, Scheithauer BW, Erickson BJ : Chemotherapy for advanced CNS ependymoma. J Neurooncol 45 : 61-67, 1999

12. Korshunov A, Witt $H$, Hielscher $T$, Benner A, Remke M, Ryzhova $M$, et al. : Molecular staging of intracranial ependymoma in children and adults. J Clin Oncol $28: 3182-3190,2010$

13. Leeper $\mathrm{H}$, Felicella MM, Walbert $\mathrm{T}$ : Recent advances in the classification and treatment of ependymomas. Curr Treat Options Oncol $18: 55$, 2017

14. Louis DN, Perry A, Reifenberger G, von Deimling A, Figarella-Branger D, Cavenee WK, et al. : The 2016 World Health Organization classification of tumors of the central nervous system: a summary. Acta Neuropathol $131: 803-820,2016$

15. Mack SC, Taylor MD : Put away your microscopes: the ependymoma molecular era has begun. Curr Opin Oncol 29 : 443-447, 2017

16. Mack SC, Witt H, Piro RM, Gu L, Zuyderduyn S, Stütz AM, et al. : Epigenomic alterations define lethal CIMP-positive ependymomas of infancy.
Nature $506:$ 445-450, 2014

17. Mansur DB, Perry A, Rajaram V, Michalski JM, Park TS, Leonard JR, et al. : Postoperative radiation therapy for grade II and III intracranial ependymoma. Int J Radiat Oncol Biol Phys 61 : 387-391, 2005

18. Massimino M, Solero CL, Garrè ML, Biassoni V, Cama A, Genitori L, et al. : Second-look surgery for ependymoma: the Italian experience. J Neurosurg Pediatr 8 : 246-250, 2011

19. Merchant TE, Li C, Xiong X, Kun LE, Boop FA, Sanford RA : Conformal radiotherapy after surgery for paediatric ependymoma: a prospective study. Lancet Oncol $10: 258-266,2009$

20. Metellus P, Guyotat J, Chinot O, Durand A, Barrie M, Giorgi R, et al. : Adult intracranial WHO grade II ependymomas: long-term outcome and prognostic factor analysis in a series of 114 patients. Neuro Oncol $12: 976$ 984, 2010

21. Modena P, Buttarelli FR, Miceli R, Piccinin E, Baldi C, Antonelli M, et al. : Predictors of outcome in an AIEOP series of childhood ependymomas: a multifactorial analysis. Neuro Oncol 14 : 1346-1356, 2012

22. Nakamura T, Fukuoka K, Ikeda J, Yoshitomi M, Udaka N, Tanoshima R, et al. : Encouraging option of multi-staged gross total resection for a C11orf-RelA fusion-positive supratentorial anaplastic ependymoma. Brain Tumor Pathol 34 : 160-164, 2017

23. Pajtler KW, Mack SC, Ramaswamy V, Smith CA, Witt H, Smith A, et al. : The current consensus on the clinical management of intracranial ependymoma and its distinct molecular variants. Acta Neuropathol 133 : 5-12, 2017

24. Pajtler KW, Pfister SM, Kool M : Molecular dissection of ependymomas. Oncoscience 2 : 827-828, 2015

25. Pajtler KW, Witt H, Sill M, Jones DT, Hovestadt V, Kratochwil F, et al. : Molecular classification of ependymal tumors across all CNS compartments, histopathological grades, and age groups. Cancer Cell 27 : 728 743, 2015

26. Panwalkar P, Clark J, Ramaswamy V, Hawes D, Yang F, Dunham C, et al. : Immunohistochemical analysis of $\mathrm{H} 3 \mathrm{~K} 27 \mathrm{me} 3$ demonstrates global reduction in group-A childhood posterior fossa ependymoma and is a powerful predictor of outcome. Acta Neuropathol 134 : 705-714, 2017

27. Parker M, Mohankumar KM, Punchihewa C, Weinlich R, Dalton JD, Li Y, et al. : C11orf95-RELA fusions drive oncogenic NF-KB signalling in ependymoma. Nature $506:$ 451-455, 2014

28. Paulino AC: The local field in infratentorial ependymoma: does the entire posterior fossa need to be treated? Int J Radiat Oncol Biol Phys 49 : 757-761, 2001

29. Paulino $A C$, Wen $B C$ : The significance of radiotherapy treatment duration in intracranial ependymoma. Int J Radiat Oncol Biol Phys 47 : 585 589, 2000

30. Ramaswamy V, Hielscher T, Mack SC, Lassaletta A, Lin T, Pajtler KW, et al. : Therapeutic impact of cytoreductive surgery and irradiation of posterior fossa ependymoma in the molecular era: a retrospective multicohort analysis. J Clin Oncol 34 : 2468-2477, 2016

31. Rudà R, Bosa C, Magistrello M, Franchino F, Pellerino A, Fiano V, et al. : Temozolomide as salvage treatment for recurrent intracranial ependymomas of the adult: a retrospective study. Neuro Oncol 18 : 261-268, 2016 
32. Venkatramani R, Dhall G, Patel M, Grimm J, Hawkins C, McComb G, et al. : Supratentorial ependymoma in children: to observe or to treat following gross total resection? Pediatr Blood Cancer 58 : 380-383, 2012

33. Venkatramani R, Ji L, Lasky J, Haley K, Judkins A, Zhou S, et al. : Outcome of infants and young children with newly diagnosed ependymoma treated on the "Head Start" III prospective clinical trial. J Neurooncol 113 : 285291, 2013
34. Vera-Bolanos E, Aldape K, Yuan Y, Wu J, Wani K, Necesito-Reyes MJ, et al. : Clinical course and progression-free survival of adult intracranial and spinal ependymoma patients. Neuro Oncol 17 : 440-447, 2015

35. Witt $H$, Mack SC, Ryzhova M, Bender S, Sill M, Isserlin R, et al. : Delineation of two clinically and molecularly distinct subgroups of posterior fossa ependymoma. Cancer Cell 20 : 143-157, 2011 